\title{
PENSION FUND MODEL DESIGN AND STATE ESTIMATION
}

\author{
Miroslav Šimandl, Marek Lešek and Ondřej Straka
}

\author{
Department of Cybernetics \\ University of West Bohemia in Pilsen \\ Univerzitní 8, 30614 Pilsen, Czech Republic \\ e-mail: simandl@kky.zcu.cz,marek.lesek@quick.cz, \\ straka30@kky.zcu.cz
}

\begin{abstract}
A design of the mathematical model of the contribution-defined pension fund is treated. The design respects the specific character and economical rules of supplementary pension system in the Czech Republic. The state of the new mathematical model is partly immeasurable. The immeasurable part of the state is estimated by the extended Kalman filter. The mathematical model and the designed estimator are illustrated with real data from a pension fund. Copyright (C)2005 IFAC
\end{abstract}

Keywords: pension funds, mathematical model, state estimation, extended Kalman Filter, stochastic system

\section{INTRODUCTION}

The purpose of this paper is to present a design and state estimation of a non-linear mathematical model of the contribution-defined supplementary pension fund in the Czech Republic.

Since the pension funds were established, the mathematical models in the UK, Canada and the USA have been designed. The pension funds can be modeled by the actuarial cost methods (traditional approach) or the dynamical models (modern approach). The traditional methods are described in Trowbridge (1952). The modern methods for benefit-defined pension funds are presented in Dufrese (1986) and Haberman and Gerrard (1996) and for contribution-defined pension funds in Vigna and Haberman (2001) and Josa-Fombellida and Rincón-Zapatero (2001).

As the Czech supplementary pension system was established by the law (Ministry of Internal Affairs, 1994 and 1999), it was necessary to provide mathematical modeling of the pension funds. The above mentioned methods use stationary state of pension fund. However, the Czech Republic supplementary pension system is growing. So a new mathematical model that takes into consideration the specifics of the Czech's pension system has to be designed.

A simplified mathematical model of supplementary pension fund was introduced in Šimandl and Lešek (2003) where the optimal investment strategies were solved.

This paper is focused on design and utilization of a pension fund mathematical model that is created in conformity with the Czech law.

The paper is organized as follows. Section 2 is devoted to design of a mathematical model of a contribution-defined pension fund, which is based on the Czech law (Ministry of Internal Affairs, 1994 and 1999). Content of Section 3 is focused on a state estimation by the extended Kalman filter. In the first part a structure of the extended Kalman Filter is introduced and in the 
second part the extended Kalman filter is used for state estimation of the pension fund mathematical model. In Section 4 a numerical example is presented and a brief summary is done in Section 5.

\section{DESIGN OF MATHEMATICAL MODEL OF PENSION FUND}

The design of the mathematical model of the pension fund is based on knowledge of the Czech law (Ministry of Internal Affairs, 1994 and 1999) and economical rules. The main presumptions for the design of the pension fund mathematical model are:

- the model is created by the law number 42/1994 Coll. wording in force

- all contributions, benefits and movement of clients are done at the beginning of each year

- assets of pension fund are evaluated by investments - an evaluation of assets is assets' pay-off minus fund costs

According to the presumptions a discrete stochastic non-linear state-space system $\mathbf{S}$ is chosen:

$$
\begin{aligned}
\mathbf{S}: \mathbf{x}(t+1) & =\mathbf{f}(\mathbf{x}(t), \mathbf{u}(t), t)+\mathbf{w}(t) \\
\mathbf{y}(t) & =\mathbf{h}(\mathbf{x}(t), t)+\mathbf{v}(t)
\end{aligned}
$$

where $\mathbf{x}(t)$ is the state vector, $\mathbf{f}(\mathbf{x}(t), \mathbf{u}(t), t)$ is known vector non-linear function called the transition function, $\mathbf{u}(t)$ is the input vector, $\mathbf{w}(t)$ is white noise, $\mathbf{y}(t)$ is the output vector, $\mathbf{h}(\mathbf{x}(t), t)$ is known non-linear function called the measuring function and $\mathbf{v}(t)$ is white noise.

Firstly, it is necessary to design a state vector $\mathbf{x}(t)$ of the pension fund model. The dimension and definition of state vector are chosen as the minimal requirement to model the real supplementary pension fund. The following form of the state vector of the pension fund mathematical model is supposed:

$$
\mathbf{x}(t)=\left[x_{1}(t), x_{2}(t), x_{3}(t), x_{4}(t), x_{5}(t), x_{6}(t)\right]^{T},
$$

where $x_{1}(t)$ is a ratio of clients who keep in pension fund at time $t+1$ (except new incoming clients) to number of clients of pension fund at time $t$ where $x_{1}(t) \in\langle 0,1\rangle, x_{2}(t)$ is an average value of all benefits per a client, $x_{3}(t)$ is an average value of contribution per client (including government support), $x_{4}(t)$ is number of all clients in the pension fund, $x_{5}(t)$ represents total assets of the pension fund and $x_{6}(t)$ is a rate of return of whole pension fund in interval $\langle t, t+1\rangle$ where $x_{6}(t) \in\langle-1, \infty)$.

The dynamical evolution of the state components in the mathematical model is based on real economical rules of the pension fund. The equations define existing relations between number of clients and assets of the pension fund.

$$
\begin{aligned}
x_{1}(t+1)= & x_{1}(t)+w_{1}(t) \\
x_{2}(t+1)= & x_{2}(t)+w_{2}(t) \\
x_{3}(t+1)= & x_{3}(t)+w_{3}(t) \\
x_{4}(t+1)= & x_{1}(t) x_{4}(t)+u_{k}(t) \\
x_{5}(t+1)= & \left(1+x_{6}(t)\right)\left[\left(x_{3}(t)-x_{2}(t)\right) x_{4}(t)\right. \\
& \left.+x_{5}(t)+u_{p}(t)\right] \\
x_{6}(t+1)= & x_{6}(t)+w_{6}(t)
\end{aligned}
$$

The equations (3),(4), (5) and (8) model an evolution of $x_{1}(t), x_{2}(t), x_{3}(t)$ and $x_{6}(t)$ as a Wiener's process. The states can be modeled hereby because the states $x_{1}(t+1), x_{2}(t+1), x_{3}(t+1)$, $x_{6}(t+1)$ depend only on the previous values of the states $x_{1}(t), x_{2}(t), x_{3}(t), x_{6}(t)$ and on values of the respective random variables $w_{1}(t), w_{2}(t), w_{3}(t)$, $w_{6}(t)$. The random variables $w_{1}(t), w_{2}(t), w_{3}(t)$ and $w_{6}(t)$ are defined as the random variables with mean $\mu_{i}$ and variance $\sigma_{i}^{2}$ where $i=1,2,3,6$.

The equation (6) represents dynamical development of the number of the pension fund clients, where $u_{k}(t)=u_{k_{1}}(t)+u_{k_{2}}(t)$ is the number of the clients that join to the pension fund at time $t$ and $u_{k_{1}}(t)$ is the amount of the new client who have not contributed yet and $u_{k_{2}}(t)$ is the number of the clients who have contributed to another pension fund and have already saved some money.

The equation (7) describes dynamical evolution of the size of the pension fund assets, where $u_{p}(t)$ is the amount which clients bring to the pension fund from other pension fund in which they have already saved.

The output equation (2) is designed so that the states $x_{2}(t), x_{3}(t), x_{4}(t)$ and $x_{5}(t)$ are directly measurable. Hence, the output equation is linear and the vector $\mathbf{v}(t)$ has the following form: $\mathbf{v}(t)=$ 0 for all $t$. Then

$$
\mathbf{y}(t)=\mathbf{C} \cdot \mathbf{x}(t)
$$

where $C$ is in form:

$$
\mathbf{C}=\left[\begin{array}{llllll}
0 & 1 & 0 & 0 & 0 & 0 \\
0 & 0 & 1 & 0 & 0 & 0 \\
0 & 0 & 0 & 1 & 0 & 0 \\
0 & 0 & 0 & 0 & 1 & 0
\end{array}\right]
$$

The system equation (1) is described by the equations (3)-(8) in detail. The output equation (2) is expanded by the equation (9).

The mathematical model of the pension supplementary fund in the Czech Republic was designed according to the Czech law (Ministry of Internal Affairs, 1994 and 1999). This model should respect all specifics of the Czech pension system. 


\section{STATE ESTIMATION BY THE EXTENDED KALMAN FILTER}

For prediction of the pension fund it is necessary to estimate the immeasurable part of the state vector. As the mathematical model of pension fund is non-linear we have to use a non-linear estimator.

During last thirty years a lot of non-linear filters were designed. They can be divided to two main groups: to global and local (Sorenson, 1974). The global filters are characterized by validity of estimates in the form of a probability density function in whole state space while local filters provide point estimates only. The list of filters is presented in Kulhavý (1996) and Söderström (1994). There are three main approaches to design the global analytical filters for non-linear stochastic systems. The analytical approach based on the model linearization and Gaussian sum approximation of probability density function Sorenson and Alspach (1971) and Šimandl and Královec (2000), the numerical approach to solution of the Bayesian recursive relations leading to the grid base filters Bucy and Senne (1971) and Šimandl et al. (2002) and the simulation approach using the Monte Carlo approximation Liu and Chen (1998) and Šimandl and Soukup (2002). These approaches generate probability density function as a result of the estimation. They have heavy computational demands for complex systems. As the defined mathematical model of pension fund results from economical reality and there has been prior information about variables $x_{1}(t) \ldots x_{6}(t)$, it is possible to use a simple non-linear filter. In this case the extended Kalman filter has been chosen (Anderson and Moore, 1979).

Application of the extended Kalman filter to model $(3)-(9)$ is shown in the following part.

Consider the stochastic system

$$
\begin{aligned}
\mathbf{x}(t+1) & =\mathbf{f}(\mathbf{x}(t), \mathbf{u}(t), t)+\mathbf{w}(t) \\
\mathbf{y}(t) & =\mathbf{C x}(t)
\end{aligned}
$$

where the vector function $\mathbf{f}(\mathbf{x}(t), \mathbf{u}(t), t)$ is given by the equations (3) to (8) and it was defined as

$$
\mathbf{f}(\mathbf{x}(t), \mathbf{u}(t), t)=\left[\begin{array}{c}
f_{1}(\mathbf{x}(t), \mathbf{u}(t), t) \\
f_{2}(\mathbf{x}(t), \mathbf{u}(t), t) \\
f_{3}(\mathbf{x}(t), \mathbf{u}(t), t) \\
f_{4}(\mathbf{x}(t), \mathbf{u}(t), t) \\
f_{5}(\mathbf{x}(t), \mathbf{u}(t), t) \\
f_{6}(\mathbf{x}(t), \mathbf{u}(t), t)
\end{array}\right]=
$$

$$
\left[\begin{array}{c}
x_{1}(t) \\
x_{2}(t) \\
x_{3}(t) \\
x_{1}(t) x_{4}(t)+u_{k}(t) \\
\left(1+x_{6}(t)\right)\left[\left(x_{3}(t)-x_{2}(t)\right) x_{4}(t)+\ldots\right. \\
\left.\ldots+x_{5}(t)+u_{p}(t)\right] \\
x_{6}(t)
\end{array}\right]
$$

The initial state $\mathbf{x}(0)$ and the white noise $\mathbf{w}_{t}$ are supposed to be Gaussian

$$
\begin{aligned}
& p(\mathbf{x}(0))=N\left(\mathbf{x}(0): \hat{\mathbf{x}}^{\prime}(0), \mathbf{P}^{\prime}(0)\right) \\
& p(\mathbf{w}(t))=N(\mathbf{w}(t): 0, \mathbf{Q}(t))
\end{aligned}
$$

and independent for all time instants $t$.

On one hand for the defined system it is easy to evaluate the approximate filtering probability density function $p_{A}\left(\mathbf{x}(t) \mid \mathbf{y}^{t}\right)$ because the output equation (12) is linear:

$$
\begin{aligned}
p_{A}\left(\mathbf{x}(t) \mid \mathbf{y}^{t}\right)= & N(\mathbf{x}(t): \hat{\mathbf{x}}(t), \mathbf{P}(t)) \\
\hat{\mathbf{x}}(t)= & \hat{\mathbf{x}}^{\prime}(t)+\mathbf{P}^{\prime}(t) \mathbf{C}^{T}\left[\mathbf{C P}^{\prime}(t) \mathbf{C}^{T}\right]^{-1} \\
& \cdot\left[\mathbf{y}(t)-\mathbf{C} \hat{\mathbf{x}}^{\prime}(t)\right] \\
\mathbf{P}(t)= & \mathbf{P}^{\prime}(t)-\mathbf{P}^{\prime}(t) \mathbf{C}^{T}\left[\mathbf{C P}^{\prime}(t) \mathbf{C}^{T}\right]^{-1} \\
& . \mathbf{C P}^{\prime}(t)
\end{aligned}
$$

where $\mathbf{y}^{t} \triangleq[\mathbf{y}(0), \mathbf{y}(1), \ldots \mathbf{y}(t)]^{T}, \hat{\mathbf{x}}^{\prime}(t)$ is mean and $\mathbf{P}^{\prime}(t)$ is covariance matrix of the approximate one step predictive probability density function $p_{A}\left(\mathbf{x}(t) \mid \mathbf{y}^{t-1}\right), \hat{\mathbf{x}}(t)$ is mean and $\mathbf{P}(t)$ is covariance matrix of the approximate filtering probability density function $p_{A}\left(\mathbf{x}(t) \mid \mathbf{y}^{t}\right)$.

On the other hand the system equation (11) is non-linear and it has to be linearized. The Taylor expansion is used to linearize equation on the surrounding of the optimal state estimation $\hat{\mathbf{x}}(t)$.

$$
\begin{aligned}
\mathbf{f}(\mathbf{x}(t), \mathbf{u}(t), t) \simeq & \mathbf{f}(\hat{\mathbf{x}}(t), \mathbf{u}(t), t)+\mathbf{F}(\hat{\mathbf{x}}(t), \mathbf{u}(t), t) \\
& (\mathbf{x}(t)-\hat{\mathbf{x}}(t))
\end{aligned}
$$

where

$$
\begin{aligned}
& \mathbf{F}(t)(\hat{\mathbf{x}}(t))=\frac{\partial \mathbf{f}(\mathbf{x}(t), t)}{\partial \mathbf{x}(t)} \mid \hat{\mathbf{x}}(t)= \\
& {\left[\begin{array}{cccccc}
1 & 0 & 0 & \hat{x}_{4} & 0 & 0 \\
0 & 1 & 0 & 0 & -\hat{x}_{4}\left(\hat{x}_{6}+1\right) & 0 \\
0 & 0 & 1 & 0 & \hat{x}_{4}\left(\hat{x}_{6}+1\right) & 0 \\
0 & 0 & 0 & \hat{x}_{1} & \left(1+\hat{x}_{6}\right)\left(\hat{x}_{3}-\hat{x}_{2}\right) & 0 \\
0 & 0 & 0 & 0 & \left(1+\hat{x}_{6}\right) & 0 \\
0 & 0 & 0 & 0 & \left(\hat{x}_{3}-\hat{x}_{2}\right) \hat{x}_{4}+\hat{x}_{5}+u_{p}
\end{array}\right]^{T}}
\end{aligned}
$$

It is possible to evaluate an approximate prediction probability density function for linearized system equation (11):

$p_{A}\left(\mathbf{x}(t+1) \mid \mathbf{y}^{t}\right)=N(\mathbf{x}(t+1):$ 


$$
\begin{gathered}
\left.: \hat{\mathbf{x}}^{\prime}(t+1), \mathbf{P}^{\prime}(t+1)\right) \\
\hat{\mathbf{x}}^{\prime}(t+1)=\mathbf{f}(\hat{\mathbf{x}}(t), t) \\
\mathbf{P}^{\prime}(t+1)=\mathbf{F}(\hat{\mathbf{x}}(t), t) \mathbf{P}(t) \mathbf{F}^{T}(\hat{\mathbf{x}}(t), t)+\mathbf{Q}(t) .
\end{gathered}
$$

The filter that was just brought out is defined by the equations (16) and (18) and is called the extended Kalman Filter.

The random variables are modeled by the Gaussian probability density function, but the values of the estimated states $x_{1}(t)$ and $x_{6}(t)$ are valid only within certain ranges. This combination causes that the values of the estimated states could be out of theirs ranges. So we have to apply two holders which keep estimated output values within theirs boundaries. The first holder keeps value of the state $x_{1}(t)$ between 0 and 1 because it represents a ratio of clients, who keep in pension fund at time $t+1$ (except new incoming clients) to number of clients of pension fund at time $t$. The second low-pass holder is used for the state $x_{6}(t)$. It holds that the value of the estimated state $x_{6}(t)$ is higher then -1 because it represents the rates of return of whole the pension fund in time interval $\langle t, t+1\rangle$.

\section{NUMERICAL EXAMPLE}

In this section a numerical example of an estimation immeasurable part of the state by the extended Kalman Filter is given.

The estimation was performed for the Military Open Pension Fund of the Czech Republic. The data was obtained from official pension fund sources and from The Association of Pension Funds of the Czech Republic (APF CR). The data up to year 2001 were used. The data contain information about the clients who have supplementary pension insurance according the original version of law (Ministry of Internal Affairs, 1994 and 1999) but also about the clients who have supplementary pension insurance according the amending act valid from the $1^{\text {st }}$ of January 2000 .

In this illustration example it is supposed that nobody comes to pension fund when he contributes to another pension fund. It means that the value of the incoming money from the other pension fund $u_{p}(t)$ is zero and the numbers of the client who have contributed to other pension funds $u_{k_{2}}(t)$ equals to zero. The input data are given in Table 1. The table contains all information about the input variable $u_{k}(t)$ and the measurable states $x_{2}(t), x_{3}(t), x_{4}(t)$ and $x_{5}(t)$.

The number of clients $y_{3}(t)$ and pension fund assets $y_{4}(t)$ are presented in Figures 1 and 2, respectively because these are the main characteristics of the pension fund.
Table 1. Input values for state estimation of the mathematical model by the extended Kalman filter (inputs $y_{1}(t)$, $y_{2}(t)$ and $y_{4}(t)$ are in thousands of CZK)

\begin{tabular}{lcccc}
\hline & \multicolumn{4}{c}{ Year } \\
\cline { 2 - 5 } & 1994 & 1995 & 1996 & 1997 \\
\hline$y_{1}(t)$ & 0 & 0.0232 & 0.3092 & 1.0378 \\
$y_{2}(t)$ & 5.4480 & 7.8120 & 7.2072 & 5.1278 \\
$y_{3}(t)$ & 0 & 95000 & 226000 & 236000 \\
$y_{4}(t)$ & 135100 & 111020 & 2263000 & 3289100 \\
$u_{k_{1}}(t)$ & 95000 & 147150 & 64240 & 41920 \\
\hline \multicolumn{5}{c}{ Year } \\
\cline { 2 - 5 } & 1998 & 1999 & 2000 \\
\hline$y_{1}(t)$ & 2.7794 & 6.7292 & 10.3200 & 15.1749 \\
$y_{2}(t)$ & 4.7798 & 7.8984 & 7.2654 & 6.9248 \\
$y_{3}(t)$ & 226000 & 217000 & 313000 & 331000 \\
$y_{4}(t)$ & 4476500 & 7068400 & 7625118 & 8742000 \\
$u_{k_{1}}(t)$ & 94960 & 119870 & 46170 & 37340 \\
\hline
\end{tabular}

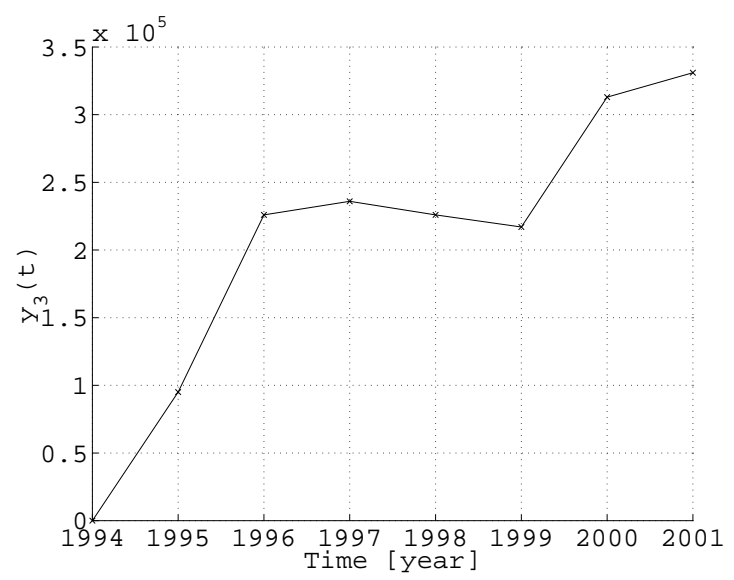

Fig. 1. Number of the clients in the pension fund $y_{3}(t)$

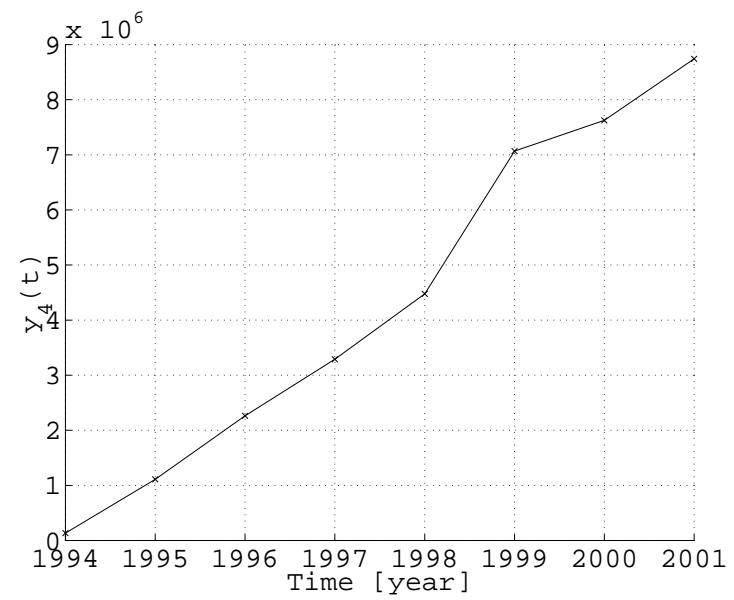

Fig. 2. The pension fund assets $y_{4}(t)$ (in thousands of $\mathrm{CZK}$ )

The initial state estimate $\hat{\mathbf{x}}_{0}^{\prime}$ was set for this numerical example to

$$
\hat{\mathbf{x}}^{\prime}(0)=\left[0, y_{1}(0), y_{2}(0), y_{3}(0), y_{4}(0), 0\right]^{T} \text {. }
$$

The variance of the initial state estimates $\mathbf{P}_{0}^{\prime}$ and variance $\mathbf{Q}_{t}$ of the white noise $\mathbf{w}_{k}$ were chosen as diagonal matrix in following form: 


$$
\begin{aligned}
& \mathbf{P}_{0}^{\prime}=\operatorname{diag}([1,0.01,0.01,0.01,0.01,1]) \\
& \mathbf{Q}_{t}=\mathbf{Q}=\operatorname{diag}([1,1000,1000,0,0,1]),
\end{aligned}
$$

where $\operatorname{diag}(\mathbf{c})$ means a diagonal matrix with vector $\mathbf{c}$ as its diagonal.

The extended Kalman filter was used for estimation of the states $x_{1}(t)$ and $x_{6}(t)$. Final results of state estimation by the extended Kalman filter are given in Table 2 . The results are presented for better orientation in figures of estimated and real values; Figure 3 for the state $x_{1}(t)$ and Figure 4 for the state $x_{6}(t)$.

Table 2. Comparison of real and estimated values for the immeasurable states $x_{1}(t)$ and $x_{6}(t)$

\begin{tabular}{lcccc}
\hline & \multicolumn{4}{c}{ Year } \\
\cline { 2 - 5 } & 1994 & 1995 & 1996 & 1997 \\
\hline$x_{1}(t)$ & 0 & 0.8300 & 0.7600 & 0.7800 \\
$\hat{x}_{1}(t)$ & 0 & 0 & 1.0000 & 1.0000 \\
$x_{6}(t)$ & 0.1160 & 0.0945 & 0.1000 & 0.1003 \\
$\hat{x}_{6}(t)$ & 0 & 0.7218 & 0.0223 & -0.0139 \\
\hline \multicolumn{5}{c}{ Year } \\
\cline { 2 - 5 } & 1998 & 1999 & 2000 & 2001 \\
\hline$x_{1}(t)$ & 0.5400 & 0.8900 & 0.9100 & 0.8600 \\
$\hat{x}_{1}(t)$ & 0.9576 & 0.9602 & 1.0000 & 0.9243 \\
$x_{6}(t)$ & 0.0970 & 0.0670 & 0.0410 & 0.0425 \\
$\hat{x}_{6}(t)$ & 0.0052 & 0.1015 & 0.0041 & 0.0311 \\
\hline
\end{tabular}

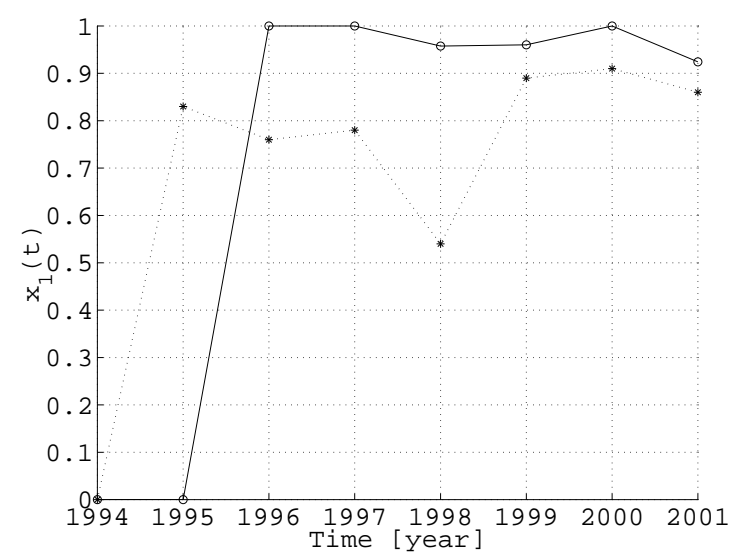

Fig. 3. Ratio of clients, who keep in pension fund at time $t+1$ (except new incoming clients) to number of clients of pension fund at time $t$ $x_{1}(t) ;(* \cdots)$ real value, $(\mathrm{o}-)$ estimated value

Results of the numerical example are reviewed for each estimated state separately because there is no economical or physical influence between the state $x_{1}(t)$ and the state $x_{6}(t)$.

Firstly, estimation of the $x_{1}(t)$, that describes an evolution of a ratio between the clients, who keep in the pension fund at time $t+1$ (except new incoming clients) and clients of the pension fund at time $t$ is performed. As it can be seen in Figure 3 and Table 2, the estimated values are very close to the physical limitation of the state $x_{1}(t)$ which is defined in Section 2 (the value of $x_{1}(t)$ must be between 0 and 1$)$. The rules for

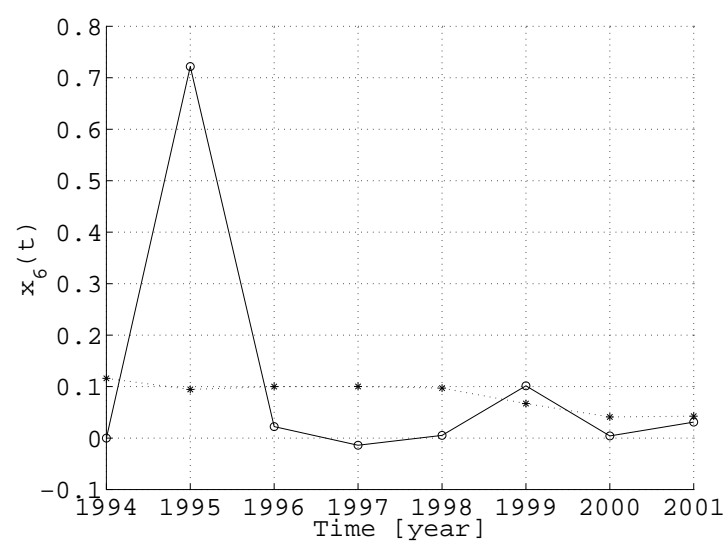

Fig. 4. Pension fund assets rates of returns $x_{6}(t)$; $(* \cdots)$ real value, $\left(\mathrm{o}_{-}\right)$estimated value

reducing the estimates of the extended Kalman filter must be applied here. It is also necessary to mention that the real values are very close to the upper bound of their limitation. Due to these facts the extended Kalman filter cannot tune itself quickly enough. So the estimated values and the real values of the state $x_{1}(t)$ are shifted for these input data. The last values of the estimated state $\hat{x}_{1}(t)$ follow the trends of real value of $x_{1}(t)$. It is expected that the filter is close to be tuned-up and the values of the state $\hat{x}_{1}(t)$ will be estimated more accurately for greater range of input data.

Figure 4 contains an evolution of real and estimated value of the pension fund rates of returns $x_{6}(t)$ for contribution-defined pension fund. It can be seen in Figure 4 and Table 2 that the estimated state $\hat{x}_{6}(t)$ oscillates around the real values of state $x_{6}(t)$ with diminishing amplitude. It is necessary to take into account that input variables are not stable and we have only eight measured variables to use. The last values of the estimated state $\hat{x}_{6}(t)$ are close to the real values $x_{6}(t)$. This fact shows that the extended Kalman filter is able to tune-up itself and yields quality estimations of immeasurable variables in a few steps.

Although real values of the variables $x_{1}(t)$ and $x_{6}(t)$ seem to be close to constant and estimations $\hat{x}_{1}(t)$ and $\hat{x}_{6}(t)$ oscillate around them, it is necessary to note that the pension fund is still in process of growth. It is clear from Figure 1 and Figure 2. The characteristics of real values will change after the market saturation in the future. Then the extended Kalman filter will adapt itself faster than the simple methods using approximation by the polynomials.

The different behaviour of immeasurable states $\hat{x}_{1}(t)$ and $\hat{x}_{6}(t)$ estimated by the extended Kalman filter lies in their limitations and complexity of the equations which describe them. 


\section{CONCLUSION}

This paper dealt with design and utilization of the mathematical model of the contribution-defined pension fund in the Czech Republic. The new mathematical model of pension fund has been derived from the Czech law (Ministry of Internal Affairs, 1994 and 1999). The technique of mathematical modelling of a pension fund may be useful even for other countries where pension funds are going to be launched.

The extended Kalman filter was applied to the mathematical model of contribution defined pension fund. The numerical example used the real data from the pension fund, which participates in the Czech pension funds market. With respect to the numerical example the results the extended Kalman filter were acceptable for use in the state estimation of the pension fund mathematical model. The values of the states $x_{1}(t)$ and $x_{6}(t)$ were estimated well and the differences between the estimated and the real value were caused by the additional holders and the range of data. The estimated values of the immeasurable state $x_{1}(t)$ were shifted to real value because the holder was used to keep the values in their defined physical limits. Influence of the holder decreased with number of applied input data. The estimated values of the second immeasurable state $x_{6}(t)$ oscillated around the real values. The amplitude decreased with increased time.

The estimation of the immeasurable states would require a long history of input data because the extended Kalman filter had to be tuned-up. The number of required input data depends on the complexity of the equations that describe the dynamic evolution of the pension fund mathematical model. The longer history of input data would yield better results.

\section{ACKNOWLEDGEMENT}

The work was supported by the Ministry of Education, Youth and Sports of the Czech Republic, project No. MSM 235200004 and by the Grant Agency of the Czech Republic, project GACR $102 / 05 / 2075$.

\section{REFERENCES}

Anderson, B.D.O. and S.B. Moore (1979). Optimal filtering. Prentice Hall. New Jersey.

Bucy, R.S. and K.D. Senne (1971). Digital Synthesis of Non-linear Filters. Automatica 7, 287298.

Dufrese, D. (1986). The dynamics of pension funding. PhD thesis. The City University, School of Actuarial Science and Statistic, London, UK.

Haberman, S. and R. Gerrard (1996). Stability of pension systems when gains/losses are amortized and rates of return are autoregressive. Insurance: Mathematics and Economics 18, 59-71.

Josa-Fombellida, R. and J.P. Rincón-Zapatero (2001). Minimalization of risks in pension funding by means of contributions and portfolio selection. Insurance: Mathematics and Economics 29, 35-45.

Kulhavý, R. (1996). Recursive nonlinear estimation. Vol. 216. Springer-Verlag. London, UK. Lecture Notes in Control and Information Sciences.

Liu, J.S. and R. Chen (1998). Sequential Monte Carlo methods for dynamic systems. J. Amer. Statist. Assoc. 93(443), 1032-1044.

Ministry of Internal Affairs (1994 and 1999). The law number 42/1994 Coll. (amend by the law number 170/1999 Coll.). Collection of Laws of the Czech republic. Prague. (in Czech).

Söderström, R. (1994). Discrete-Time Stochastic Systems, Ser. Systems and Control Engineering. Prentice Hall. Upper Saddle River, NJ.

Sorenson, H. (1974). On the developtment of practical nonlinear filters. Inform. Sci. 7, 230-270.

Sorenson, H.W. and D.L. Alspach (1971). Recursive Bayesian estimation using Gaussian sums. Automatica 7, 465-479.

Trowbridge, C.L. (1952). Fundamentals of pension funding. Transactions of the Society of Actuaries $4,17-43$.

Vigna, E. and S. Haberman (2001). Optimal investment strategy for defined cotribution pension schemes. Insurance: Mathematics and Economics 28, 233-262.

Šimandl, M. and J. Královec (2000). Filtering, Prediction and Smoothing with Gaussian Sum Representation. In: SYSID 2000: Proceedings of Symposium on System Identification. IFAC, Elsevier Science. Santa Barbara, California, USA.

Šimandl, M. and M. Lešek (2003). Controlling of pension fund investment by using Bellman's Optimality Principle. In: Proceedings of IFAC conference Control System Design (CDS'03). IFAC, Elsevier Science. Bratislava, The Slovak Republic.

Šimandl, M. and T. Soukup (2002). Simulation Monte Carlo methods in Extended Stochastic Volatility Models. Int. Journal of Intelligent Systems in Accounting, Finance $\&$ Management 11, 109-117.

Šimandl, M., J. Královec and T. Söderström (2002). Anticipative Grid Design in PointMass Aproach to Non-linear state estimation. IEEE Transactions Automatic control vol. 47 no. 4, 699-702. 